Results Median age of pts was 41 (range 20-79) yrs. Total lifetime yrs on anti-TNFs was 740.6 (331.2 on Ifx, 409.4 on $\mathrm{Al}$ ) yrs. 133 pts were treated with Ifx (75 females), 4 had previously been on Al. Median length of Rx with Ifx was 20 (range 1 dosestill on at 140$)$ months. $98(73.6 \%)$ pts on Ifx were on a concomitant immunomodulator drug. 141 pts were treated with $\mathrm{Al}$ (79 females), 53 had previously been on Ifx. Median length of Rx with Al was 33 (range <1-still on at 89) months. 62 (44.0\%) pts on $\mathrm{Al}$ were on a concomitant immunomodulator.

$54(40.6 \%)$ pts had AEs whilst on Ifx (see Table 1 for severity), including lymphoma (2 pts), solid organ tumours (3), pulmonary TB (2), infusion reaction (13), cutaneous side effects (SEs) (7), other infections (12). 33 pts were still on Ifx at the time of this study. 30 pts (22.6\%) stopped Ifx due to AEs. 13 pts had an infusion reaction. Pts were most likely to have an infusion reaction at infusion 2 (6 pts).

Overall, 57 (39.7\%) pts suffered from AEs on Al (see Table 1 for severity), including solid organ tumours (2 pts), cutaneous SEs (10), neurological symptoms (3) and other viral/bacterial infections (28). 88 pts were still on $\mathrm{Al}$ at the time of this study. 21 pts (14.9\%) stopped $\mathrm{Al}$ due to AEs.

Pts were more likely to suffer from an AE with increasing age ( $p=0.041$ for Ifx, $\mathrm{p}=0.016$ for $\mathrm{Al}$ ). Patients over 50 yrs were more likely to suffer from an $\mathrm{AE}$ than those less than 50 ( $\mathrm{p}=$ 0.015 for Ifx, $\mathrm{p}=0.015$ for $\mathrm{Al}$ ). Pts over 70 yrs were more likely to suffer from a moderate or severe $\mathrm{AE}$ on $\mathrm{Al}$ ( $\mathrm{p}=$ 0.009), there was no relationship for Ifx. Gender, smoking status and use of immunomodulators had no effect on AEs. No significant relationship found between length of Rx and development of AEs. No statistically significant difference found in AEs frequency between If $x$ and $\mathrm{Al}$.

Conclusion This study found that AEs are independent of the length of time on anti-TNFs, but are associated with increasing age of the patient. Patients over 50 yrs are more likely to have an $\mathrm{AE}$ on Ifx and Al. Patients over 70 yrs are more likely to have a moderate or severe $\mathrm{AE}$ on $\mathrm{Al}$.

Disclosure of Interest None Declared.

\section{PTU-070 NON-ADHERENCE WITH RECTAL PREPARATIONS IN COLITIC PATIENTS. FACT OR FICTION?}

B Kok*, A Waters, A Jenkins. Department of Gastroenterology, Princess Royal University Hospital, King's College Hospital NHS Foundation Trust, London, UK

\subsection{6/gutjnl-2014-307263.144}

Introduction Rectal preparations containing steroids or 5-aminosalicylates are an effective treatment for distal colitis. Nonetheless, it is perceived that this therapy is associated with poor levels of adherence and that patients are not readily accepting of it. This might make gastroenterologists reluctant to prescribe rectal preparations. The patient experience and factors determining adherence with this modality of treatment have not been investigated before. The aim of our survey is to evaluate patient compliance with rectal preparations and explore the possible reasons why compliance is not always achieved.

Methods A short anonymised survey was distributed over a 3month period at general gastroenterology and IBD outpatient clinics to patients with a known diagnosis of ulcerative colitis. There were questions on baseline demographics and other details relating to compliance. A free text option was also available for suggestions that could improve compliance.

Results Over the period November 2013-January 2014, a total of 59 completed surveys were received. 21 of 59 patients $(36 \%)$ had never been prescribed a rectal preparation. Of the remaining 38 patients with reported experience of rectal preparations, $60 \%$ were male with a median age of 45 years. Surprisingly, $76 \%$ of these (29 patients) answered 'Yes' to being compliant with the preparation (s), and they had been prescribed courses ranging from once/day for 2 weeks to thrice/day long-term. 20 patients had been prescribed foam and/or liquid enemas, 8 patients a suppository, and 1 patient a suppository and foam enema. 93\% of the compliant patients reported an improvement in their symptoms with the use of rectal preparations. Though compliant, $24 \%$ reported 'Yes' to having difficulties using the rectal preparation citing "insertion difficulties" and "difficulty retaining the fluid" and "at work" as the main reasons. When asked for suggestions to improve compliance, $62 \%$ felt reduction of treatment frequency to once/day would help, and $21 \%$ felt that better explanation about administration would help. Of the 9 patients who admitted to being noncompliant, 3 had difficulties with administration, 2 experienced pain, and the majority felt that a better explanation about administration would have improved compliance. As expected, the majority of noncompliant patients felt that the rectal preparation had made no difference to their symptoms.

Conclusion Compliance with prescribed rectal preparations is surprisingly high. This is an encouragement to continue promoting its use. Compliance could be further improved by allowing for patient factors such as work hours and adjusting dosing times, and by dedicating time to explain how the medication should be administered.

Disclosure of Interest None Declared.

\section{PTU-071 METHOTREXATE THERAPY FOR ULCERATIVE COLITIS IN THE DISTRICT GENERAL HOSPITAL SETTING: A USEFUL SECOND-LINE OPTION FOR PATIENTS}

C Hammond*, BR Disney, A Xiarchou, D Chohan, J Coyle, G Wood, S Raman, E Sung. Gastroenterology, George Eliot Hospital, Nuneaton, UK

\subsection{6/gutjnl-2014-307263.145}

Introduction The case for methotrexate (MTX) therapy has been comprehensively compiled in Crohn's disease, with trials showing its efficacy in both induction and maintenance of remission. By contrast, the evidence remains patchy for ulcerative colitis (UC). BSG guidelines recommend its trial as a second-line agent in those patients intolerant of, or resistant to, azathioprine (AZA) or mercaptopurine (MP). Previous studies show conflicting results for MTX in UC; with response rates between 22$33 \%$, with a high rate of colectomy, $44 \%$, in one of the studies. We aimed to review the clinical effectiveness of MTX in our cohort of UC patients who had previously tried, or failed, with thiopurine therapy.

Methods A retrospective analysis of patients taking MTX for UC was carried out. Subjects were identified from our inflammatory bowel disease database. All patients had trialled thiopurine therapy prior to MTX. Their outcomes on these treatments, including reasons for discontinuation, were recorded. It is current policy within our department to start with oral MTX (plus folate supplementation), initially $15 \mathrm{mg}$ once weekly, increasing to $20 \mathrm{mg}$ or $25 \mathrm{mg}$ as necessary. Clinical response at 12 weeks and 12 months was used to assess efficacy of MTX treatment. The primary endpoint was steroid-free remission.

Results A total of 21 UC patients (male $=62 \%$ ) were identified. Median age was 61 years (range 21-82). Disease pattern was extensive (43\%), left-sided (19\%), and recto-sigmoid (38\%). 